\title{
Identifying the long-term care beneficiaries: Differences between risk factors of nursing homes and community-based services admissions
}

\section{BACKGROUND}

The widespread increase in life expectancy is perhaps one of the greatest achievements of humankind. Consequently, changes in demographic features - characterized by an elderly population, an increase of chronic diseases and new patterns of growing morbidity and functional restrictions [1,2] - are already a reality in most developed countries.

Thus, the evolution and (re)configuration of the health system should be influenced by the adoption of adequate health policies in order to encourage the coordination of the healthcare, social support and long-term care (LTC) sectors as a whole. As for the LTC sector, it embraces all forms of continuous, personal, rehabilitation and nursing care, designed to provide assistance over prolonged periods to people with mental and physical impairment and unable to look after themselves without some degree of external support [3,4]. Often, this care provision is associated with two main settings: (1) institutional, known as Nursing Homes (NH); and (2) non-institutional, known as Home and Community-Based Services (HCBS).

Although these settings of care seem to offer similar services, they differ in terms of frequency, intensity and degree of supervision $[3,4]$. Thus, several differences between the two settings have been reported in the literature, either related to expenditures [58], functional changes [9-14], mortality [6,15], quality of life $[5,16]$ or frequency of acute care visits $[6,13,15]$. Nevertheless, as mentioned in two literature reviews [4,13], given the differences in data collection, selection of populations and methodological strategies, there are very few studies from which to draw conclusions about the differences in effectiveness between HCBS and $\mathrm{NH}$ care in several of these outcomes.

It has been widely mentioned in the literature that patients prefer to stay in their homes for as long as possible in order to keep their social networks and to maintain their family environment [15-19]; nevertheless, when care needs are extensive, admission to an institution may be inevitable and necessary [19]. For that matter, in order to (re)define new policies to determine the proper setting of care for each person, much attention has been devoted to identifying the main risk factors leading to institutionalization $[4,20,21]$. Usually, those include patients' characteristics such as: (1) socio-demographic characteristics, (2) medical conditions, and especially (3) the physical and cognitive dependence levels.

Regarding the first group of risk factors, the association between education level and the use of LTC services is not well proven [20-23], while characteristics such as older age $[6,8,14,19,20,22,24-27]$, female gender $[6,7,12,14,19,20,24,26-28]$ and being widowed or single $[17-20,22,23,27]$ are usually known to be risk factors contributing for patients being institutionalized. As for the social network, the deeper the family/caregiver involvement, the more likely that an individual remains in the community (HCBS) $[7,8,12-$ $14,17,21,22]$.

Concerning the influence of chronical medical conditions, the findings are inconsistent. A literature review found low evidence of significant differences in several conditions (e.g. stroke, hypertension, respiratory diseases, incontinence or depression) as a 
predictor of a $\mathrm{NH}$ admission [21]. A study from Germany concluded that individuals receiving HCBS care had a higher burden of diseases [6], while other authors found a higher prevalence of dementia, Parkinson's disease, diabetes, renal disease or cancer in $\mathrm{NH}$ populations, when compared with those receiving home care $[14,19,20,23,27]$.

Individuals' dependence levels cannot be directly extrapolated from their clinical diagnosis, therefore a more detailed assessment aimed at identifying physical and cognitive limitations needs to be conducted. Such evaluation contributes to the definition of each care plan either for maintaining or delaying the total or partial loss of capacities. Based on several studies from the US [7,11,20,22,23,25], Canada [19,27], Taiwan [5], South Korea [28], Germany [6,17,18,21], The Netherlands [26] and a study including eight European countries [8], the conclusions were very similar: those receiving care at $\mathrm{NHs}$ are usually cognitively and physically more dependent than those receiving HCBS.

Finally, although other risk factors may be associated to $\mathrm{NH} / \mathrm{HCBS}$ placement, such as region where the care is provided [14,16,22,26,27], expected length of care $[7,14,15,24,25]$, referral entity and placement process [26], their influence is not yet well proven.

\section{The Portuguese long-term care system}

In Portugal, the National Network for Long-term Integrated Care (Rede Nacional de Cuidados Continuados Integrados) was created in 2006 as a partnership between the Ministry of Health and the Ministry of Employment and Social Solidarity, taking advantage of the already existing resources (largely non-profit-making institutions) [29].

As described elsewhere [30], the type of care provided is organized into the two main settings of care mentioned earlier, namely $\mathrm{HCBS}$ and $\mathrm{NH}$, with this last being provided through three types of care units [29]: Convalescence Units (Unidades de Convalescença, UC), Medium-Term and Rehabilitation Units (Unidades de Média Duração e Reabilitação, UMDR) and Long-Term and Maintenance Units (Unidades de Longa Duração e Manutenção, ULDM).

Regarding the placement process, after individuals are referred from a hospital or a primary care unit, the care request is sent to the Local Coordination Teams (Equipas de Coordenação Local, ECL), and then, if the individual fulfils the requirements, the request is directed to the Regional Coordination Teams (Equipas de Coordenação Regional, ECR). To streamline the coordination between the referring entity and the individual's admission in any setting of care, the national guideline recommends that potential LTC beneficiaries should be flagged in the first 72 hours after this type of care need is identified, especially during hospitalization.

\section{OBJECTIVES}

The main goal of this work is twofold: 1) to identify the main risk factors on the likelihood that an individual is placed in each setting of care; and 2) to explore to what extent the populations placed into NH (UC, UMDR and ULDM) and HCBS differ from each other. We expect to contribute to improve the knowledge about the LTC in Portugal, its placement process, settings of care and beneficiaries' characteristics. 
To the best of the authors' knowledge, this is likely to be the first study to explore and draw conclusions from the Portuguese dataset of LTC about risk factors for admissions in the existing care settings available in the country.

\section{METHODS}

\section{Data source}

We used the national database from the Portuguese LTC monitoring system [31], which gathers the information collected by staff of all settings of care and is used to develop the care plan for each patient. The data used was provided by the Portuguese Central Administration of Health System, entity responsible for managing the Portuguese longterm care system. Before sharing the data, all patients' identifiers, such as patients' identification in each setting of care and patient record number were anonymized. Variables like name, social security number, phone number or address are not shared with researchers.

The dataset had information for 27,832 patients, however, 1,924 were without information regarding gender, marital status and family/neighbour support, 1,777 were receiving palliative care, 951 were without information regarding their cognitive/physical status and 2,196 were less than 60 years old. Thus, we were left with completed information about 20,984 individuals, aged 60 years old or more, admitted and discharged in 2015, in Portugal mainland. For each individual in the dataset, besides the variables providing information about setting of care, region of care unit, referral entity, placement process and admission/discharge dates, we also considered information collected by the instrument used in Portugal to identify the dependence level of each individual in three main dimensions: biological, psychological and social (more information on the instrument can be found in Lopes and colleagues (2018)).

Assessment of the overall cognitive and physical dependence levels of a patient is as follows. In first place, each individual is scored according to his/her ability to answer the questions regarding orientation and to perform each of the activities of daily living considered [31,32]. Scores range from 0 to 3 depending on the level of dependence of the individuals: 0 - bad/incapable, when the individual is not able to cooperate and needs indispensable and regular caregivers and/or means of support; 1 unsatisfactory/dependent, when the individual can cooperate but needs indispensable and regular caregivers and/or means of support; 2 - satisfactory/autonomous, when the individual cooperates but needs regular means (but not caregivers) of support; 3 good/independent, when the individual does not need caregivers and/or means of support. The physical overall status is determined by the lowest score obtained in the eight activities of daily living, while the cognitive status results from the average of the scores in the ten orientation questions. Based on these two scores, each individual's overall physical and cognitive status is then assigned into one of four dependence groups, from bad/incapable to good/independent.

\section{Statistical analysis}

Concerning the descriptive analysis, several variables were taken into account to identify the main differences between the populations in each setting of care (Table 1). Given the importance of ensuring the shortest time in the placement process, three phases were analysed: (1) time taken to notify the request for care between the ECL and the ECR; (2) time between the arrival of the request at ECR and the individual's admission; 
and (3) total time elapsed between the arrival of the request at ECL and the individuals' admission in each setting of care.

Using the information collected by staff to identify the dependence level in performing each cognitive/physical activity, considering the scores ranging from 0 (bad/incapable) to 3 (good/independent), we used the One-Way ANOVA to identify and compare the mean dependence scores at admission between settings of care, in each cognitive/physical activity.

Finally, in order to identify the main risk factors on the likelihood that an individual receives care in each setting of care, two methods were used. Firstly, a logistic regression with Forward-LR method was performed, using the two main settings of care as the dependent variable. Secondly, since the three $\mathrm{NH}$ units of care follow an intrinsic order as the individuals' dependence level increases, the ordered logistic regression model was used to estimate the probability of a patient with certain characteristics to be placed in one of the three $\mathrm{NH}$ units. Thus, since the Unit with the highest predicted probability ('NH Unit Expected') it is assumed to be the most appropriate for the patient according to the chosen model, we compared the Unit where he/she was admitted ('NH Unit Observed') and the expected Unit given by the model ('NH Unit Expected'). In both of these analyses, the marginal effects were calculated.

\section{Explanatory variables}

In the two final analyses, three main sets of explanatory variables were included: sociodemographic characteristics such as age, gender, marital status, family/neighbour support, and educational level; medical conditions; and dependence levels such as cognitive and physical status at admission. The choice of variables is not only based on the factors mentioned in the literature to be significantly associated with the risk of being admitted to a $\mathrm{NH}$, but also considering the several features already gathered in the national database from the Portuguese LTC monitoring system.

\section{Covariates}

As for covariates, we used variables external to patient characteristics, such as referral entity (hospital versus others), region of care to account for differences of care supply and length of placement process (days between ECL to Admission).

All analyses were run with SPSS Statistics software (v.20, IBM SPSS, Chicago, IL) and with STATA statistical software (release 13), with a significance level of 0.05 .

\section{RESULTS}

\section{Descriptive analysis}

There are substantial differences between the numbers of individuals treated in each Portuguese geographic region, with three of them (North, Centre and Lisbon and Tagus Valley) encompassing $80 \%$ of the analysed patients (Table 1). When compared to the HCBS population, the $\mathrm{NH}$ individuals present, on average, a similar age (80 years old), a higher percentage of females but a lower percentage of married people, family/neighbour support and illiterate individuals. The diseases of the circulatory system, injury and poisoning, neoplasms and diseases of the musculoskeletal system are responsible for $70 \%$ of the total population medical conditions $(64 \%$ and $72 \%$ for 
HCBS and NH population, respectively). As for the cognitive and physical status at admission, the percentage of individuals classified into the two lowest levels is higher at $\mathrm{NH}(54.5 \%$ and $71.2 \%)$ than in HCBS (45.9\% and $69.0 \%)$ setting of care. Regarding the referral entities, although overall $64 \%$ of individuals were referred from hospitals, most people referred to HCBS (56\%) came from other entities, especially from the primary care centres, while this happens only in $27 \%$ of the cases for $\mathrm{NH}$. Regarding the hospital services, the internal medicine (18\%/32\%) and orthopaedic services (15\%/21\%) are the ones with the highest percentage of referral to the HCBS/NH setting of care (data not shown, available upon request). Finally, regarding the placement process and the length of care, whereas the first is longer for $\mathrm{NH}$ admissions, the individuals at HCBS receive care for a longer period.

Comparing the three $\mathrm{NH}$ units of care, the population at $\mathrm{UC}$ is slightly younger, has a higher percentage of females, fewer married people and a lower percentage of illiterate individuals than the other two units. As for the medical conditions, while the two main groups of individuals at UC and UMDR suffer from diseases of the circulatory system and injury and poisoning, almost $16 \%$ of the ULDM population presents mental disorders as their main admission diagnosis (the second largest group). Concerning the cognitive/physical status at admission, the dependence levels increase as we go from the UC to the ULDM. Finally, the percentage of individuals referred from hospitals to the $\mathrm{UC}$ is higher; the placement process is faster; and the length of care is lower when compared to both the UMDR and to the ULDM.

(Table 1)

\section{Dependence levels at admission}

In Figure 1 are depicted the results of the One-Way ANOVA regarding patients mean dependence scores at admission by setting of care for both the cognitive and the physical status. Differences between settings of care were found to be significant with $p \leq 0.001$ for all the questions included in the cognitive and physical assessment.

Although the population at $\mathrm{NH}$ presents a higher dependence level in all assessed cognitive and physical activities when compared to HCBS population (data not shown, available upon request), there are also differences within the three types of $\mathrm{NH}$. Patients' cognitive impairment at admission in all activities gets worse from UCs to ULDMs, as lower scores are associated with higher dependence levels. In this case, while patients admitted in UCs had a globally "satisfactory" (score 2) cognitive status, those receiving care in ULDMs were mainly scored as "unsatisfactory" (score 1). Moreover, results show a higher cognitive impairment on the ability to answer questions about temporal then spatial orientation. Regarding the physical status, although there are differences on the average dependence profile in performing daily activities between settings, most patients present a high level of impairment in all activities: mostly range between the status "incapable" (score 0) and "dependent" (score 1).

(Fig. 1) 


\section{Determining the risk of being admitted into an NH or HCBS setting of care}

Table 2 shows the main predictors of $\mathrm{NH}$ admission. Results show that, when compared to those receiving HCBS care, being female $(+1.3$ percentage points, p.p.), not being married (widow or single/divorced: +4.1 p.p.) or being literate $(+4.9$ p.p.) increases the probability of an individual receiving care in an $\mathrm{NH}$ setting. On the contrary, older age (0.02 p.p.) and having family/neighbour support (-12.4 p.p.) have an opposite effect. Regarding the medical conditions, the risk of being institutionalized increases with mental illness (+10.1 p.p.), diseases of the circulatory (+9.1 p.p.), nervous ( +7.5 p.p.) or musculoskeletal (+4.5 p.p.) systems. Finally, considering the influence of the dependence levels at admission, when compared to the individuals with the worst level of impairment, being classified at a higher level of cognitive and physical independence decreases the chance of being admitted into an $\mathrm{NH}$ setting of care (-6.9 p.p. and -3.6 p.p., respectively).

(Table 2)

\section{Determining the risk of being admitted into each $\mathrm{NH}$ unit}

Considering the sociodemographic and the main medical conditions, results in Table 3 show that the following factors increase the probability of being referred to an institutionalized unit of care with anticipated longer stay (ULDM): older age (+0.3 p.p.), and having neoplasms (+12.0 p.p.) or mental illness (+20.1 p.p.). On the opposite side, being literate (+2.1 p.p.), having diseases of the skin/subcutaneous tissue (+16.8 p.p.) or diseases of the musculoskeletal system (+26.6 p.p.) increase the chance of being referred to a short stay (UC) unit of care.

Of the several individuals' characteristics included in this analysis, the cognitive and physical status at admission are those that play a greater influence in determining the admission in each $\mathrm{NH}$ unit of care. Compared to those with a worse cognitive impairment, the individuals considered cognitively independent have a higher probability (+16.5 p.p.) of being referenced to the UC, whereas the probability of going to the UMDR or to the ULDM decreases in 3.6 p.p. and 12.9 p.p., respectively. Similarly, the more independent an individual is considered at admission, the less likely that individual is to be referred to a care unit with a longer expected length of care, compared to those with higher physical dependence (UC: +12.3 p.p., UMDR: -3.0 p.p. and ULDM: -9.3 p.p.).

(Table 3)

Finally, table 4 shows the mismatch between the observed placements of patients to each $\mathrm{NH}$ unit of care ("NH Unit Observed") and what would have been the expected unit ("NH Unit Expected"), taking into account all variables included in the previous analysis (Table 3). Results show that in $69 \%, 54 \%$ and $46 \%$ of the cases predictions matched the actual referred units (UC, UMDR and ULDM, respectively - shaded cells). 
(Table 4)

The discrepancies between predictions and observed placements go in several directions. For example, for more than half of the patients referred to ULMDs the model expects referral to units where more intensive care is provided, namely $9 \%$ to UC and $46 \%$ to UMDR. As for the patients admitted into UCs, the model predicts that $29 \%$ could have benefited from less intensive care provided in UMDRs. Finally, for the patients initially admitted into UMDRs, according to the model $28 \%$ should have been receiving more intensive care in UCs and $18 \%$ less intensive care in ULDMs.

\section{DISCUSSION}

\section{Dependence levels at admission}

Given its importance in the LTC context, several authors have assessed patients' ability to perform single activities, either comparing the mean number of dependencies between institutionalized and non-institutionalized populations [11,21,23], assessing its correlation with the overall functional status [28] or using them as explanatory variables to predict the probability of being admitted to an $\mathrm{NH}$ setting [18].

In this study, after identifying the mean dependence level scores at admission in each activity, two major conclusion arise. First, the level of cognitive impairment is higher on the ability to answer questions about temporal rather than spatial orientation. Second, most patients present a high level of impairment in performing all physical activities. Thus, the identification of patients' dependence in each activity, whether physical or cognitive, should be used by staff members to direct their efforts towards the recovery of the ability to perform those activities with the highest levels of disability, and to adapt the individual care plan. Moreover, it could enable policymakers to better define the staff mix in each setting of care, according to patients' needs.

\section{Determining the risk of being admitted into an NH or HCBS setting of care}

This study found that the main risk factors significantly associated with $\mathrm{NH}$ admission were the following: being female, having low social support (being widowed/single/divorced and having a low family/neighbour support), being literate, presenting some specific medical conditions (mental illness, diseases of the nervous, the circulatory and the musculoskeletal system) and being physically/cognitively dependent.

Regarding the influence of gender and low social support, this study reaches conclusions similar to those of previous researchers $[6,8,17-22,24,26-28]$. The fact that women have a higher life expectancy, and at the same time higher prevalence of physical and mental health comorbidities [2], implies that the absence or the death of a spouse could aggravate the level of dependence and the inability to take care of themselves, and consequently increase the chances of receiving receive institutionalized care [17]. Thus, not only is essential to define joint policies between the social and healthcare sectors in order to identify these risk groups, but also to create appropriate responses which enables them to stay in the community as long as possible with quality of life.

For what concerns the effects of age, the evidence of this study is opposite to that of several previous works [6-8,12,14,17,19-22,25-27], having found that older age has a 
small but positive influence on the chance of receiving HCBS care. Since older age is not a referral criterion for LTC admissions in Portugal, it might be that these individuals have more family/caregiver support, which may contribute to delaying the admission into an $\mathrm{NH}$ facility. On the other hand, the fact that older age decreases the risk of being admitted into short stay units may be explained by the fact that these units were created for individuals with a greater potential for autonomy recovery. Even though the evidence points to physical rehabilitation being associated with physical function improvement in LTC facilities $[9,10]$ and even considering that in general younger individuals may be better able to regain some of the lost autonomy, still more research is needed to confirm the effectiveness of care, including follow-up studies.

As for medical conditions, the findings are inconsistent. Although there is evidence that $\mathrm{NH}$ residents have usually a higher burden of diseases [19,20,23,27], and also that chronic diseases such as depression, incontinence or diabetes are significant risk factors of $\mathrm{NH}$ admission [21], recent studies concluded that no statistically significant differences are present between institutionalized and non-institutionalized individuals, after adjusting for the individuals' baseline characteristics [12,14,15]. Unfortunately, it is not possible to identify the burden of diseases of the Portuguese LTC individuals, due to the lack of information besides the main diagnosis at admission. Nevertheless, the finding that some medical conditions are a significant risk factor of $\mathrm{NH}$ admission can help the policy makers define specific care plans to account for the different needs.

The individuals' dependence levels are often recognized as highly relevant to determine the intensity of care services and consequently the best setting of care. In line with several studies [5,8,19,25-28], we found that being classified in a higher cognitive and physical dependence level increases the risk of $\mathrm{NH}$ admission. Considering these findings, two reflections are necessary: first, it is essential that policy makers pay attention to staffing mix in order to tailor each setting of care with the proper resources to meet the individuals' needs; additionally, there is a need to change the financing model, by including patients' dependence levels and risk adjustment models, in order to avoid adverse selection of patients based on their case-mix by each setting of care.

\section{Determining the risk of being admitted into each $\mathrm{NH}$ unit}

Finally, a striking result is the low proportion of correct matches between the "Observed" versus the "Expected" $\mathrm{NH}$ unit of care. In fact, only $69 \%, 54 \%$ and $46 \%$ of the individuals were correctly predicted to be referred to the UC, UMDR and ULDM, respectively, after adjusting for several variables. It is crucial to underline that any mismatch between predicted and observed outcome is not necessarily evidence of a poor screening process; it simply signals cases where the placement decision is driven not only by the considered observable variables, but also by additional unobservable factors or constraints. This confirms that the placement process presents a high complexity and several challenges which cannot easily be simplified through a model based on the considered variables alone. On the other hand, the still high number of cases where the model matches the observed unit of care confirms the validity of the model for the majority of cases.

Although recent studies have found differences in results after controlling for baselines characteristics $[7,12,14,15]$, our findings may be a consequence of several unobserved factors, which may have been taken into account in the placement process. Examples could include: (1) the patient's family will; (2) geographical constraints in terms of family 
access/distance from unit; (3) care supply availability at time of placement; (4) lack of $\mathrm{NH}$ units of care and regional asymmetries in the provision of care in Portugal, and so on.

As a proxy for the (higher) complexity of some placement processes, the length of placement process until admission was considered; this captured in part the effect of some of the unobserved factors, whose presence in a process may have delayed the final placement decision or the actual admission to the unit of care.

Besides the fact that $93 \%$ of the Portuguese population had poor access to institutionalized care, given the lack of beds available [33], our results may suggest an inadequate placements of individuals, inefficient use of scarce resources, as well as the care provision not being appropriate for the real needs. However, in the future recording some of the abovementioned additional factors could clarify whether this is actually the case, and improve the decision-making process by helping understand what other determinants (if any) should be taken into consideration throughout the placement decision.

\section{Limitations}

Despite the many important findings of this study, several limitations should be pointed out. Firstly, differences in data collection, selection of populations and methodological strategies across studies make it difficult to compare results, as highlighted in several literature reviews $[4,10,13,16,21]$. Secondly, these results are difficult to compare to other national studies, given that this database is still underexplored, and that there are virtually no studies in the literature since the creation of the Portuguese LTC system. Thirdly, given the scarce information regarding medical conditions, it was not possible to identify and assess the comorbidity burden of the individuals in each setting of care. Finally, additional variables that may influence the overall results - such as the ability to perform more complex activities (e.g. instrumental activities of daily living), transitions between settings of care, or other risk factors (like smoking, obesity, alcohol and drugs) - were not analysed due to their poor quality. Further research to understand the influence of these variables is required.

\section{CONCLUSIONS}

Based on this study, several conclusions arise. To the best of the authors' knowledge, in comparison to other studies published, the approximately 21,000 patients included here represent the largest dataset analysed for a period of 1 year. Having said this, the results here presented are robust for the Portuguese LTC context.

Furthermore, it is important to take into account variations in the needs for individual assistance, avoiding similar situations as the one found in this study, whereby patients with the same dependence level are placed in different settings of care and end up receiving different levels of care. The admission of patients into care units not suited for their existing needs, either in terms of under-care or over-care, represent a waste of scarce resources. With these results, policy-makers are provided with evidence enabling them to better plan future investments in the LTC network.

Thirdly, since the region of care influences the setting where a patient is placed, it may be a consequences of differences in care supply across the country. Thus, the existence 
of a multidisciplinary case management team may be a key factor to balance both demand and supply needs, in order to guarantee an appropriate use of resources. Moreover, there is a need to implement an auditing process to ensure continuous improvement in the placement process. Nevertheless, further research is needed to assess the extent of regional asymmetries in order to tailor the care supply in each region to the needs of its population.

This study contributes to the literature by shedding light on the differences between the $\mathrm{NH}$ and the HCBS populations and by identifying the main predictors of admission in each type of care. Furthermore, results are analysed separately for three types of $\mathrm{NH}$ units, responsible for providing care to individuals with different dependence levels. Last, but not least, although the placement process is highly complex, presents several challenges and is also influenced by additional unobservable factors or constraints, this study contributes for a better understanding of the mismatches in the admission process occurring in the Portuguese LTC and provides leads to what can be improved.

\section{ACKNOWLEDGEMENTS}

We want to acknowledge the Portuguese Central Administration of Health System for providing the information registered by the Integrated Bio-psychosocial Assessment Instrument. Author NR gratefully acknowledges financial support from FCT (Project CEMAPRE - UID/MULTI/00491/2013 financed by FCT/MCTES through national funds).

\section{REFERENCES}

[1] Rechel B, Grundy E, Robine J-M, Cylus J, Mackenbach JP, Knai C, et al. Ageing in the European Union. Lancet 2013;381:1312-22. doi:10.1016/S0140-6736(12)62087-X.

[2] Barnett K, Mercer SW, Norbury M, Watt G, Wyke S, Guthrie B. Epidemiology of multimorbidity and implications for health care, research, and medical education: a cross-sectional study. Lancet 2012;380:37-43. doi:10.1016/S0140-6736(12)60240-2.

[3] Kaye HS, Harrington C. Long-term services and supports in the community: Toward a research agenda. Disabil Health J 2015;8:3-8. doi:10.1016/j.dhjo.2014.09.003.

[4] Wysocki A, Butler M, Kane RL, Kane RA, Shippee T, Sainfort F. Long-Term Services and Supports for Older Adults: A Review of Home and Community-Based Services Versus Institutional Care. J Aging Soc Policy 2015;27:255-79. doi:10.1080/08959420.2015.1024545.

[5] Kuo Y-C, Lan C-F, Chen L-K, Lan VM. Dementia care costs and the patient's quality of life (QoL) in Taiwan: home versus institutional care services. Arch Gerontol Geriatr 2010;51:159-63. doi:10.1016/j.archger.2009.10.001.

[6] Schwarzkopf L, Menn P, Leidl R, Graessel E, Holle R. Are community-living and institutionalized dementia patients cared for differently? Evidence on service utilization and costs of care from German insurance claims data. BMC Health Serv Res 2013;13:2. doi:10.1186/1472-6963-13-2.

[7] Newcomer RJ, Ko M, Kang T, Harrington C, Hulett D, Bindman AB. Health Care Expenditures After Initiating Long-term Services and Supports in the Community Versus in a Nursing Facility. Med Care 2016;54:221-8. doi:10.1097/MLR.0000000000000491.

[8] Wübker A, Zwakhalen SMG, Challis D, Suhonen R, Karlsson S, Zabalegui A, et al. Costs of care for people with dementia just before and after nursing home placement: primary data from eight European countries. Eur J Heal Econ 2015;16:689-707. doi:10.1007/s10198-014-0620-6. 
[9] Forster A, Lambley R, Young JB. Is physical rehabilitation for older people in long-term care effective? Findings from a systematic review. Age Ageing 2010;39:169-75. doi:10.1093/ageing/afp247.

[10] Crocker T, Young J, Forster A, Brown L, Ozer S, Greenwood DC. The effect of physical rehabilitation on activities of daily living in older residents of long-term care facilities: systematic review with meta-analysis. Age Ageing 2013;42:682-8. doi:10.1093/ageing/aft133.

[11] Kane RL, Lum TY, Kane RA, Homyak P, Parashuram S, Wysocki A. Does Home-and Community-Based Care Affect Nursing Home Use? J Aging Soc Policy 2013;25:146-60. doi:10.1080/08959420.2013.766069.

[12] Lee TW, Cho E, Yim ES, Lee HS, Ko YK, Kim BN, et al. Activities of Daily Living in Nursing Home and Home Care Settings: A Retrospective 1-Year Cohort Study. J Am Med Dir Assoc 2015;16:114-9. doi:10.1016/j.jamda.2014.07.013.

[13] O'Caoimh R, Cornally N, Weathers E, O'Sullivan R, Fitzgerald C, Orfila F, et al. Risk prediction in the community: A systematic review of case-finding instruments that predict adverse healthcare outcomes in community-dwelling older adults. Maturitas 2015;82:321. doi:10.1016/j.maturitas.2015.03.009.

[14] Lee K, Cho E. Activities of daily living and rehabilitation needs for older adults with a stroke: A comparison of home care and nursing home care. Japan J Nurs Sci 2016;14:103-11. doi:10.1111/jjns.12139.

[15] Blackburn J, Locher JL, Kilgore ML. Comparison of Long-term Care in Nursing Homes Versus Home Health: Costs and Outcomes in Alabama. Gerontologist 2016;56:215-21. doi:10.1093/geront/gnu021.

[16] Toot S, Swinson T, Devine M, Challis D, Orrell M. Causes of nursing home placement for older people with dementia: a systematic review and meta-analysis. Int Psychogeriatrics 2017;29:195-208. doi:10.1017/S1041610216001654.

[17] Luppa M, Riedel-Heller SG, Luck T, Wiese B, van den Bussche H, Haller F, et al. Agerelated predictors of institutionalization: results of the German study on ageing, cognition and dementia in primary care patients (AgeCoDe). Soc Psychiatry Psychiatr Epidemiol 2012;47:263-70. doi:10.1007/s00127-010-0333-9.

[18] Hajek A, Brettschneider C, Lange C, Posselt T, Wiese B, Steinmann S, et al. Longitudinal Predictors of Institutionalization in Old Age. PLoS One 2015;10:e0144203. doi:10.1371/journal.pone.0144203.

[19] Bartfay E, Bartfay WJ, Gorey KM. Dementia care in Ontario, Canada: evidence of more timely diagnosis among persons with dementia receiving care at home compared with residential facilities. Public Health 2016;130:6-12. doi:10.1016/j.puhe.2015.10.002.

[20] Gaugler JE, Duval S, Anderson KA, Kane RL. Predicting nursing home admission in the U.S: a meta-analysis. BMC Geriatr 2007;7:13. doi:10.1186/1471-2318-7-13.

[21] Luppa M, Luck T, Weyerer S, Konig H-H, Brahler E, Riedel-Heller SG. Prediction of institutionalization in the elderly. A systematic review. Age Ageing 2010;39:31-8. doi:10.1093/ageing/afp202.

[22] Muramatsu N, Yin H, Campbell RT, Hoyem RL, Jacob MA, Ross CO. Risk of nursing home admission among older americans: does states' spending on home- and community-based services matter? J Gerontol B Psychol Sci Soc Sci 2007;62:S169-78.

[23] Wieland D, Boland R, Baskins J, Kinosian B. Five-year survival in a program of allinclusive care for elderly compared with alternative institutional and home-and community-based care. Journals Gerontol - Ser A Biol Sci Med Sci 2010;65 A:721-6. doi:10.1093/gerona/glq040. 
[24] Häcker J, Hackmann T. Los(t) in long-term care: empirical evidence from German data 2000-2009. Health Econ 2012;21:1427-43. doi:10.1002/hec.1805.

[25] Gruber-Baldini AL, Stuart B, Zuckerman IH, Simoni-Wastila L, Miller R. Treatment of dementia in community-dwelling and institutionalized medicare beneficiaries. J Am Geriatr Soc 2007;55:1508-16. doi:10.1111/j.1532-5415.2007.01387.x.

[26] Helvik AS, Skancke RH, Selbæk G, Engedal K. Nursing home admission during the first year after hospitalization - The contribution of cognitive impairment. PLoS One 2014;9. doi:10.1371/journal.pone.0086116.

[27] Penning MJ, Cloutier DS, Nuernberger K, MacDonald SWS, Taylor D. Long-term Care Trajectories in Canadian Context: Patterns and Predictors of Publicly Funded Care. J Gerontol B Psychol Sci Soc Sci 2016:gbw104. doi:10.1093/geronb/gbw104.

[28] Kim YH, Kwon $\mathrm{CH}$, Shin HI. Validity of motor impairment scale in long-term care insurance system of Korea. Ann Rehabil Med 2013;37:403-12. doi:10.5535/arm.2013.37.3.403.

[29] D.R. Decree-Law 101/2006. D.R. I Serie A, 6th of July - Creation of the Portuguese National Network for Long-term Care [in Portuguese] 2006:3856-65. http://www.acss.min-saude.pt/wp-content/uploads/2016/10/Decreto-Lei_101_2006-1.pdf.

[30] Lopes H, Mateus C, Hernández-Quevedo C. Ten Years since the 2006 Creation of the Portuguese National Network for Long-Term Care: Achievements and Challenges. Health Policy (New York) 2018;122:210-6. doi:10.1016/J.HEALTHPOL.2018.01.001.

[31] Abreu Nogueira J, Girão M, Guerreiro I. Post Acute and Long Term Care: instrument for evaluating outcomes National Network for Integrated Continuous Care Portugal. Lisbon: UMCCI; 2010.

[32] Botelho MA. Autonomia Funcional em Idosos: Caracterização multidimensional em idosos utentes de um centro de saúde urbano (Functional Autonomy in Elders: Multidimensional characterization in elderly users of an urban primary care centre). Faculdade de Ciências Médicas da Universidade Nova de Lisboa, 1999.

[33] ERS. Access, quality and competition in long-term and palliative care [in Portuguese]. Porto: 2015. 